\title{
RESEARCH ON WHEAT DISEASES AND INSECT PESTS GEOGRAPHIC INFORMATION SYSTEM
}

\author{
Hongyan Shen ${ }^{1}$, Gang Liu ${ }^{1}$ and Yinbing $\mathrm{Ge}^{2}$ \\ ${ }^{I}$ China Agricultural University, Beijing, P.R. China shy0907@hotmail.com pac@acau.edu.cn \\ ${ }^{2}$ Currently study in Utah State University, Logan, UT, USA geyinbing@hotmail.com
}

\begin{abstract}
A geographical information system for wheat diseases and insect pests is developed, which can provide valuable information for farmers to appropriately prevent diseases and insect pests so as to decrease the environment pollution, using the techniques of inference engine, BP network, etc.
\end{abstract}

Key words: GIS、 inference engine、BP Network、ODBC

\section{INTRODUCTION}

In recent years, the technique of wheat planting has been improved. However, how to prevent wheat diseases and insect pests is still a key problem. As most farmers do not understand rules of occurrence and popularity of wheat diseases and insect pests, they cannot prevent diseases and insect pests effectively and reasonably. Even though it can increase yield and cut the throat of the diseases and insect pests to use the pesticide widely, the environment has been polluted, the natural control has been lowered and so on. These series of problems have influenced the agricultural production of our country. To guide farmers to prevent diseases and insect pests appropriately, a geographical information system for wheat diseases and insect pests is developed.

The techniques used include Geographic Information System (GIS), Expert System (ES) and Artificial Neural Network (ANN). GIS is a software 
platform used to input, save, analyze, deal with, and express geographic space data. ES is one of the most mature techniques in Artificial Intelligence (AI) area. It emphasizes the effect of the special knowledge to intelligent behavior. It simulates the ways of thinking of and digests the knowledge like a human being. ANN is a nonlinear dynamics model which possesses strong memory, self-adaptation, self-study and is capable of dealing with complicated patterns. The system modules, such as consultation, graphic display and data management, contain massive information and provide higher reliability by combining GIS with ES. ES imitates human's logical thinking and ANN imitates human's experience thinking. Therefore, the prediction-decision module in the system is not only established with experts' experiences but also can be corrected automatically.

\section{STUCTURE AND FUNCTION}

Connecting GIS, ES, and ANN with object oriented knowledge representation method, a geographical information system for wheat diseases and insect pests is developed. Based on reports of wheat diseases and insect pests, diseases and insect pests prevention and cure are systematized and GIS platform in mode of controller is linked with application. It involves weather factors, plant-protection knowledge, scientific findings, expertise and GIS with computer technology. The GIS for wheat diseases and insect pests has five function modules to applied to the wheat growth process. They are predict-decision module, diagnoses module, history disaster map showing module, consultation module, and database management. The whole structure of this system is shown in figure 1 .

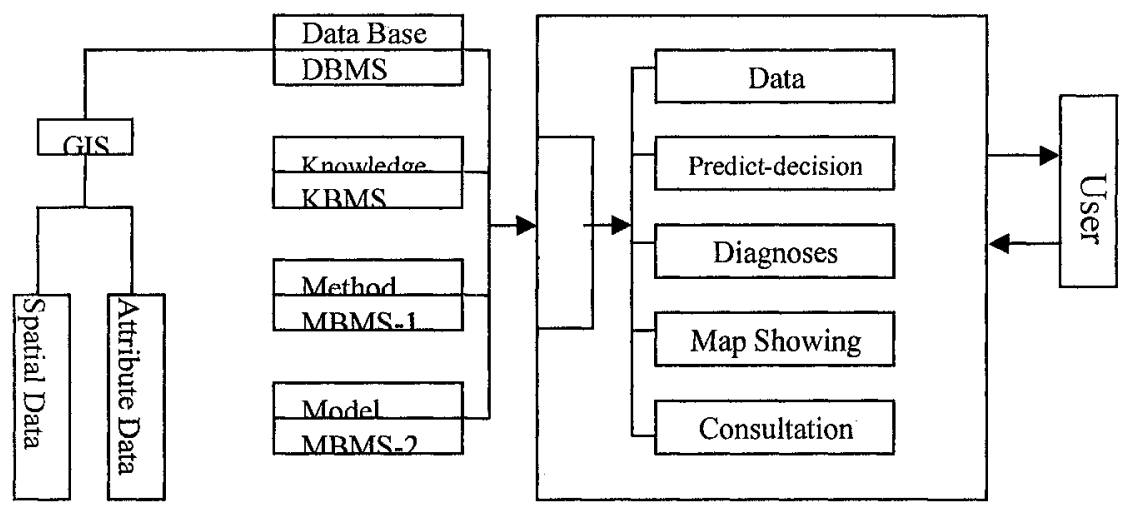

Figure 1: the Structure of the System 
In figure 1, DBMS represents database management system ; KBMS represents knowledge database management system ; MBMS-1 represents method database management system; and MBMS-2 represents model database management system .

\section{TECHNOLOGY ANALYSIS OF THE SYSTEM}

\subsection{Linking Modules with GIS}

The Loose Coupling is used to link system modules to GIS . Via the Loose Coupling, the operation module can call some functions of GIS such as data input, data edit, data display, and database management. The operation module first reads data from GIS through the interface of ODBC (Open DataBase Connectivity). ODBC is a standard access method that can access DBMS from our system. It is based on the Call-Level Interface (CLI) specifications from X/Open and ISO/IEC for database APIs and uses Structured Query Language (SQL) as its database access language. After this, the operation module calculates data and writes new data back into database of GIS using the same format.

\subsection{Inference Engine}

Inference engine is the generic control mechanism that applies the axiomatic knowledge present in the knowledge base to diseases and insect pests to arrive at some diagnosis conclusion for farmers. It is the core component of all expert systems.

\subsubsection{Inference Strategy}

The system adopts the multi-level inaccurate reasoning method. Multilevel inaccurate reasoning means that expert system obtains relatively definite conclusion and goal by the uncertain algorithm, in terms of judgment in previous period, incomplete or uncertain knowledge and data. Reasoning result is the strategy to determine object and relevant value. The process of inference is to identify the reliability. The system utilizes the inheritance of object-oriented knowledge expression method to realize multilevel and inaccurate inference, to obtain reliability of the object through distribution weight of each property and the distribution reliability of user's choices 'yes', 'no' and 'uncertainty'. 


\subsubsection{Control and Search Strategy}

The system uses Depth-First Search (DFS) algorithm. The ultimate goal is to find the answer. When the first conclusion is verified with a congruous symptom, the characteristics of this symptom need to be verified but not the others. If the characteristics are suitable, final conclusion can be made. Otherwise, other symptoms need to be verified. DFS is suitable for the inference of hierarchical structure knowledge expression. The process of inference is to calculate the reliability in terms of the user's choice. How to calculate the reliability is introduced as follows: first, choose an object; second, set the initial attribute parameter as $\mathrm{Q}_{1}$, reliability as $\mathrm{K}_{1}$, the second attribute parameter as $\mathrm{Q}_{2}$, reliability as $\mathrm{K}_{2} \ldots \ldots$.the $\mathrm{N}^{\text {th }}$ attribute parameter as $\mathrm{Q}_{\mathrm{n}}$, reliability as $\mathrm{K}_{\mathrm{n}}$; finally, the reliability of the diagnose structure is:

$$
\frac{\sum_{n} Q_{n} K_{n}}{\sum_{n} Q_{n}}
$$

the value of $\mathrm{K}_{\mathrm{n}}$ is: $K_{n}=\left\{\begin{array}{c}1 \\ 0 \\ 0.5\end{array}\right.$

if the user's choice is 'yes', $K_{n}=1$; if 'no', $K_{n}=0$; if 'uncertainty', $K_{n}=$ 0.5 .

The following is the example how to use inference engine to diagnose the wheat rust disease. First, enter the main interface of system and click consultation button. Then, follow the voice instructions of system and answer the questions ("yes", "no", or "uncertainty). Items you have chosen are immediately displayed in the list box. If the information is not what you expect, double click the items to cancel it. Thereafter, you can find the diagnosis result including the name of diseases, reliability etc. Pictures are shown on the screen of the diseases, harm character, occurrence regulations, and prevention methods. 
GEOGRAPHY INFORMATIONS SYSTEM

Table1. Diagnosis process of wheat rust diseases

\begin{tabular}{lllll}
\hline Attribute \& character & $\begin{array}{l}\text { Weighted } \\
\text { Value }\end{array}$ & Ve & Vs & Reliability \\
\hline Are wheat diseases? & 10 & Yes & Yes & 1 \\
Are Leaf diseases? & 10 & Yes & Yes & 1 \\
White mycelia spot & 20 & No & No & 0 \\
Disheveled spore \& henna & 30 & No & No & 0 \\
Disheveled spore \& puce & 30 & No & Uncertain & 0.5 \\
Disheveled spore \& orange & 30 & Yes & Yes & 1 \\
Prominent spore & 20 & Yes & Yes & 0 \\
\hline
\end{tabular}

The diagnosis reliability is 0.8 .

\subsection{BP Network}

ANN is a nonlinear dynamics simulation model of a human nervous system, which contains a collection of neuron units communicating with each other via axon connections. ANN has been applied in many fields including speech synthesis, diagnostic problems, medicine, business and finance, robotic control, signal processing, computer vision and many other problems of pattern recognition. Moreover, neural models show promise in achieving human-like performance over more traditional artificial intelligence techniques.

Currently, dozens of neural networks have been developed. The parallel network structure is adopted in BP neural network model - one of the most popular networks. BP network concludes three layers: input layer, hidden layer, and output layer. The study of BP network primarily contains four steps:

1) Input mode forward pass (input mode forwards calculation to middle layers and then to output layer): This step is to calculate the actual output corresponding to input mode. The actual output of the $t^{\text {th }}$ unit in the output layer is defined as $\mathcal{C}_{t}$

$$
c_{t}=f\left(l_{1}\right) \quad(\mathrm{t}=1,2, \ldots, \mathrm{q})
$$

where,, is the activation value of the $t^{\text {th }}$ unit in the output layer. $f$ is activation function of type $\mathrm{S}$ :

$$
f(x)=\frac{1}{1+\exp (-x)}
$$


The activation function of type $\mathrm{S}$ is closer than other types of function to the signal output form of the biology nerve center and it is consecutive and differentiable.

2) Output error backward pass: In the first step, the error between actual output and expectation is calculated. If the error goes beyond the limited value, the weights of network will be revised. It is called error backward pass because BP is revised from the back to the front. The calculation begins from the output layer to the middle layer and then to the input layer. The revised error of the output is:

$$
d_{t}^{k}=\left(y_{t}^{k}-c_{t}^{k}\right) f^{\prime}\left(l_{t}^{k}\right)
$$

where, $y_{t}{ }^{k}$ is the expectation, $c_{t}{ }^{k}$ is the actual output, $f^{\prime}($.$) is the derivative$ of the output layer function.

The revised error of each unit in middle layers is:

$$
e_{j}^{k}=\left(\sum_{t=1}^{q} v_{j t} \cdot d_{t}^{k}\right) f^{\prime}\left(s_{j}^{k}\right)_{(\mathrm{j}=1,2, \ldots, \mathrm{p} \mathrm{k}=1,2, \ldots, \mathrm{m})}
$$

It is caused by revised errors transferred from $q$ output layers. After educing the revised error, the weighted value between the output layer and the middle layers and from the middle layers to the input layer can be adjusted conversely with the values of $d_{t}^{k}$ and $e_{j}^{k}$

3) Circular memory training: Circular memory training is to make the output error of the network run to the minimum. At each training mode, the BP network should be trained circularly hundreds of times, maybe ten thousands, to remember it. This kind of circular memory training actually repeats the input mode forward pass and output error backward pass again and again.

4) Judgment of the learning result: After each circular memory training, the network should judge the learning result. The purpose of the judgment is to check whether the output error is in the limited area. If it is, the learning ends. Otherwise, the network goes on circularly training. The training is a process that the global error of the network tends to the minimum.

The initialization of BP network is to set up the topological structure (input layer, hidden layer, and the number of the nerve code in the output layer). After setting some main parameters of the BP network algorithm (learning rate, aim error, the maximum loop number, and times of display interval), the BP network starts the training. 
The prediction module in the system is built by BP network. VB language is used to design the main interface, to realize the HumanComputer Interaction function, to establish the link with Matlab at the same time. The Matlab is applied to complete the network training and prediction. Example codes are as follows:

Dim Matlab As Object

Dim MReal1 (5, 5) As Double

m MImag() As Double

SetMatlab=CreateObject("Matlab.Application")

Matlab.Execute("a=hilb(5)")

Call Matlab.GetFullMatrix("a","base", MReal1, MImag)

What the prediction function to produce and display is the grade of the diseases and insect pests in the region based on four parameters in the area: average temperature, lowest temperature, hours of sunshine, and rainfall. The module can also provide short-term prediction. The main purpose is to simulate the spread of diseases and insect pests in a short-term period and the corresponding result can be displayed in the map.

\section{CONCLUSION}

ES 、 oriented object method, ANN are utilized in this research to analyze GIS and the occurrence rules of wheat diseases and insect pests. Referring to the main diseases and insect pests, the system realizes prediction, consultation, diagnoses etc. The system not only deals with spatial data but also infers the result and makes decisions. It can transfer abstract data to compendious electronic maps and display distribution rules of the diseases and insect pests. In a word, the system aims to provide help for decreasing the cost of agricultural product, protecting environment, etc.

\section{ACKNOWLEDGEMENTS}

This program is supported by Chinese National Funded High Science and Technology Research Project (863 project) - the long-distance diagnosis platform of plant diseases and insect pests based on network 2002AA243031. 


\section{REFERENCES}

[1] Xing Xianfang. Comprehensive Administration of Crop Diseases and Insect Pests Geographic Information System [ D]. Bao Ding: Publishing Company of agricultural university of Hebei, 2004: 19-31.

[2] RAMANMC,LINERF.An expert advisory system for wheat disease management $m$ [J].Plant Disease, 1994(2):209-215.

[3] Kuang Pusheng, Jiang Wenke, Liu Gang. Precision Agricultural Elements [M]. Beijing: Publishing Company of Agricultyre University of China, 1999.30-60.

[4] He Xingui . Knowledge Disposal and Expert System. Beijing: Publishing National Defence Industry, 1990.

[5] Wu Xincai. Geographic Information System Theory and Method. Beijing: Publishing House of Electronics Industry, 2002.

[6] Zhong Xinyi ,Pan Xinan, Yang Yixian. Beijing: Intelligence Theory and Technology Artificial Intelligence and Neural Network. Publishing House of People Posting, 1992.

[7] Robert P C, Rust R H, Larson W E. Preface. Proceeding of Site Specific Management for Agriculture Systems. The 2nd International Conference on Re-search and Development Issues in Site-Specific Crop Management, 1994. 